\title{
Analogue peptides for the immunotherapy of human acute myeloid \\ leukemia
}

Susanne Hofmann ${ }^{1}$, Andrew Mead ${ }^{2}$, Aleksandrs Malinovskis ${ }^{2}$, Nicola R. Hardwick ${ }^{3,4}$, \& Barbara-ann Guinn ${ }^{2,4,5^{*}}$

${ }^{1}$ Third Clinic for Internal Medicine, University of Ulm, Ulm, Germany, ${ }^{4}$ Department of Haematological Medicine, Guy's, King's \& St. Thomas' School of Medicine, King's College London, The Rayne Institute, 123 Coldharbour Lane, London, UK ${ }^{5}$ Cancer Sciences Unit, Southampton University Hospitals Trust, University of Southampton, Southampton, UK.

Current Affiliations:

${ }^{2}$ Department of Life Sciences, University of Bedfordshire, Park Square, Luton, UK.

${ }^{3}$ Division of Translational Vaccine Research, Beckman Research Institute, City of Hope National Medical Center, Duarte, California, USA.

Correspondence should be addressed to: Dr. Barbara Guinn,

Department of Life Sciences,

University of Bedfordshire,

Park Square, Luton, LU1 3JU

UK

Tel: $\quad$ +44 1582743573

E-mail: barbara.guinn@beds.ac.uk

Keywords: analogue peptides; adult acute myeloid leukemia; clinical trials; PASD1; heteroclitic peptides; NPM1. 


\section{Abbreviations:}

AML acute myeloid leukemia

CEA carcinoembryonic antigen

CML chronic myeloid leukemia

CTA cancer-testis antigen

CTL cytotoxic T-lymphocyte

HLA human leukocyte antigen

IFA incomplete Freund's adjuvant

LAA leukemia associated antigen

LSC leukemic stem cell

MAGE melanoma associated antigen

MRD minimal residual disease

NPM1 $1^{\text {mut }} \quad$ nucleophosmin 1 gene mutation

PASD1 Per Arnt Sim Domain containing 1

pMHC peptide-major histocompatibility complex

SEREX Serological analysis of expression cDNA libraries

TCR T-cell receptor

wt wild type

WT1 Wilms' tumor gene product 1 


\begin{abstract}
The use of peptide vaccines, enhanced by adjuvants, has shown some efficacy in clinical trials. However, responses are often short-lived and rarely induce notable memory responses. The reason is that self-antigens have already been presented to the immune system as the tumor develops, leading to tolerance or some degree of host tumor cell destruction. To try to break tolerance against self-antigens, one of the methods employed has been to modify peptides at the anchor residues to enhance their ability to bind major histocompatibility complex (MHC) molecules, extending their exposure to the T-cell receptor. These modified or analogue peptides have been investigated as stimulators of the immune system in patients with different cancers with variable but sometimes notable success. In this review we describe the background and recent developments in the use of analogue peptides for the immunotherapy of acute myeloid leukemia (AML) describing knowledge useful for the application of analogue peptide treatments for other malignancies.
\end{abstract}

\title{
Précis
}

Peptide vaccines often induce short-lived immune responses. Analogue and mutated peptides have been investigated to determine whether they can stimulate improved immune responses against wild type peptides presented on tumor cells in human leukemia. 


\section{Identification and classification of tumor-associated antigens}

The rationale for $\mathrm{T}$-cell mediated cancer immunotherapy relies on the existence of tumorspecific or tumor-associated antigens (TAAs) capable of generating an antigen-specific, cytotoxic immune response (reviewed in [1-3]). The first TAA identified was melanoma associated antigen (MAGE), which was identified in the early 1990s by T-cell expression cloning [4]. However it was the subsequent development of serological analysis of expression cDNA libraries (SEREX) which allowed the rapid identification of antigens from a range of tumor types [5]. A repository detailing more than 2,000 antigens found by this technique has been developed and called the cancer immunome database (http://ludwigsun5.unil.ch/CancerImmunomeDB/).

Cheever et al. [6] used a three-step approach for decision making to (1) define features relevant for TAAs, (2) to weight those features in accordance with their practical relevance, and to (3) rank known TAAs using their weighted features. Although none of the antigens had all of the described "ideal" features, over half were immunogenic in clinical trials, and nearly one-third had suggestive clinical efficacy in the "therapeutic function" category. Cheever's list contained several leukemia associated antigens (LAAs) including Wilms' Tumor 1 (WT1) (reviewed recently in [9]), which was identified as the most suitable antigen for immunotherapy. It should be noted that the weighted features described by Cheever can also be used to compare newly identified antigens with those in the published list. However, the comparison of a solid tumor and a leukemia/hematological malignancies-only list for ranking antigens would be of interest to the research community [7], allowing reflection on the different weightings of TAAs verus LAAs with a view to their application in clinical trials for solid and liquid tumors, respectively. It appears that many of the antigens expressed 
with frequencies of note in solid tumors are not expressed frequently in patients with hematological malignancies $([7,8])$, the obvious exception being WT1.

\section{Tumor antigen targets for the vaccination of AML patients}

The antigens identified in AML (and indeed most other tumor-types) can be divided broadly into two groups with regards to the restriction of their expression in healthy tissues. The first group includes cancer-testis antigens (CTAs), so called because of an expression limited to cancer and immunologically protected tissues, such as testis and placenta, which lack MHC class I expression. Examples of these include the Per Arnt Sim Domain containing 1 (PASD1) antigen [10] and the helicase antigen (HAGE) [8]. The second grouping is that of the 'TAA' which, while expressed by tumor cells, are also found in some healthy tissues. One example is WT1 which is over-expressed by 10-100-fold in leukemia cells compared with $\mathrm{CD} 4^{+}$healthy hematopoietic progenitors. Importantly, T-cells specific for a WT1-derived peptide/human leukocyte antigen (HLA)-A2 complex, are capable of killing $\mathrm{WT}^{+}$leukemia cells but not healthy $\mathrm{WT}^{+}$cells, in vitro, indicating that a disparity in antigen expression, or the context of presentation, can be sufficient for T-cells to discriminate between leukemic cells and healthy tissue [11].

Although plenty of LAAs have been identified and several vaccination trials exist showing clinical and immunological responses, the break-through is still pending. One explanation is that the ideal target antigen has yet to be identified. In AML, the primary response to conventional chemotherapy is very good, but there is also a high risk of relapse. One reason is that the chemotherapeutic agents used are not able to eliminate chemo-resistant or quiescent leukemic stem cells (LSCs). It is possible to monitor minimal residual disease 
(MRD) by real-time PCR to determine the nucleophosmin 1-mutational (NPM1 $\left.{ }^{\mathrm{mut}}\right)$ load of AML cells [12]. WT1 represents another molecular marker for early assessment of MRD as the expression levels in normal hematopoietic progenitor cells are much lower than in leukemic cells [13]. An ideal target for vaccination would therefore be an LAA that is highly expressed in LSC as well as leukemic blasts.

\section{Characteristics of peptides used in vaccination strategies}

Exposure of a peptide epitope to the immune system without the context of the whole antigen can be a problem. Exogenously administered antigen may not follow the same processing pathway as endogenously derived antigen. There are also differences in the length of a peptide used for vaccinations. Short peptides with a length of approximately 8 to 10 amino acids can bind directly to MHC class I complexes and are presented to cytotoxic Tlymphocytes (CTLs). However peptides with a low affinity for MHC class I require de novo synthesized MHC and vesicular transport, while longer peptides always undergo classic or endosomal processing resulting in presentation, both in the context of MHC class I and II. Due to the omnipresence of MHC class I complexes on all nucleated cells, non-professional antigen-presenting cells (APCs), like fibroblasts, also present antigens to CTLs. However, fibroblasts do not express the co-stimulatory molecules needed for T-cell activation and hence, could induce immune tolerance to the presented peptide. Peptides with a length of more than 13 amino acids attract APCs which function by phagocytosis or macropinocytosis and are thus presented on MHC class II complexes.

A common criticism of vaccination strategies aimed at single epitopes is that tumor escape variants can evolve during the course of the disease. However, if vaccination results in tumor 
lysis, numerous antigens would be released and be taken up by professional APCs, which can prime responses to an extended number of antigens. This phenomenon, known as epitope spreading, was seen in a trial in breast and ovarian cancer patients vaccinated with dendritic cells pulsed with MUC-1 peptide. Vaccination resulted in the expansion of MUC-1 specific T-cells, as well as the gradual emergence of T-cells specific for carcinoembryonic antigen (CEA) and MAGE-3 [14].

There have been measurable successes with wild type (wt) peptide vaccines in clinical trials for myeloid leukemia. Several groups have developed peptide vaccines against proteinase 3, including the PR1 epitope, and/or WT1 which have led to both immunological and clinical responses against AML cells following vaccination [15-17]. However, some trials using short $\mathrm{CD}^{+}$peptides have been disappointing with one of the major limitations being short-lived immune responses due to a lack of memory T-cell induction [17-20]. The general limitations of peptide vaccines have led to investigations that focussed on the enhancement vaccine efficacy such as the use of longer peptides [21], a mixture of peptides [22] or the utilization of more complex adjuvants in terms of composition [23]. Alternatively, $\mathrm{CD}^{+}$peptide vaccines can be co-administered with class II 'helper' peptides [21], CpG-containing oligodeoxynucleotides [24] or cytokines [16,24,25].

\section{Adjuvants to enhance immunogenicity}

Simple peptide vaccines can be poorly immunogenic unless administered with a strong adjuvant [26] and oil-in-water formulations such as incomplete Freund's adjuvant (IFA) are the commonly used as vehicles for peptides in clinical trials [16,21,24,25]. In particular, the combination of $\mathrm{CpG}$ oligos with peptide vaccines appears to be very effective. A Phase I trial 
in melanoma patients, employing vaccination with a Melan-A peptide analogue and CpG in IFA, reported strong $\mathrm{CD}^{+} \mathrm{T}$-cell responses with more than $3 \%$ of circulating $\mathrm{CD}^{+}{ }^{+} \mathrm{T}$-cells being specific for Melan-A and only minor, transient side effects being reported [27]. Lower dose peptide vaccines in combination with CpG 7909 have also been shown to significantly increase degranulation and correlated with strengthened cytotoxicity responses in melanoma patients in clinical trials [28]. This is important when considered in the context of PR1 peptide vaccination trial, which reported that only patients showing at least a doubling in the number of PR1 specific T-cells had clinical responses [29].

To increase the immunogenicity of a peptide by increasing co-stimulatory molecule expression and therefore T-cell activation, several adjuvants have been investigated. Granulocyte-macrophage colony-stimulating factor (GM-CSF) and Montanide ISA-51 (a water-in-oil emulsion to slow the release of antigen) are widely used adjuvants while other adjuvants, such as toll-like receptor agonists, are also of interest [27]. Several papers suggest better vaccination efficacy would occur in an environment with decreased regulatory T-cells numbers when a single-dose cyclophosphamide [30,31] was used. However, cyclophosphamide was also shown to increase the population of myeloid-derived suppressor cells, which are able to suppress CTL cell proliferation [32]. Interestingly, DNA hypomethylation agents and histone deacetylation agents (such as Decitabine and Azazytidine) can restore MHC class I and antigen processing machinery expression [33]. Interferon (IFN)- $\gamma$ has been shown to induce MHC class I and $\beta 2$-microglobulin expression [34]. Another axis of the immune system is that of natural killer cells being activated by thalidomide or lenalidomide [35]. Thalidomide was seen to enhance IL-2 production by $\mathrm{CD}^{+}$T-cells and enhanced cyototoxicity of natural killer cells, while the tyrosine kinase 
inhibitor sunitinib was shown to decrease the circulation of myeloid derived suppressor cells and regulatory T-cells in renal cell carcinoma patients [36].

\section{Choice of peptides: affinity, crypticity and immunodominance}

Tumor antigens are often non-mutated, wt self-proteins and as such they pose a problem for immunotherapy. The immune system is constantly exposed to these antigens, and, as a result, T-cells recognising high affinity, immunodominant epitopes may be deleted in the thymus. However, T-cells recognising low affinity, subdominant epitopes, may not have been deleted [37], and if tolerance can be overcome, may be expanded by vaccination. These expanded Tcells could then target tumor cells which express cryptic epitopes. Cryptic epitopes are epitopes that are not presented, or recognized by T-cells, unless they are produced in unusually large concentrations, or are freed from the configuration of their native antigen [38]. Cryptic epitopes have been identified within several cancer associated antigens, such as HER-2/neu [39], human telomerase reverse transcriptase (hTERT)[39] and cytochrome P450 1B1 [40]. Cytochrome P450 1B1 antigen is expressed in both solid and hematological cancers [40], and was found to be capable of expanding antitumor CTLs [41].

Gross et al. found that the CTL repertoire in HHD mice (HHD mouse characterisation described in [42]) against murine telomerase reverse transcriptase (mTERT) was tolerized against high affinity epitopes [43]. In contrast T-cells specific for low affinity peptides were only mildly affected by tolerogenic control, and stimulated the expansion of high avidity, mTERT-specific CTLs. In addition, no signs of autoimmunity were detected in the vaccinated mice. The authors suggested that TERT expression in normal cells is not sufficient to stimulate T-cell responses against low affinity peptides, but is sufficient when presented in 
the context of tumor cells. This could explain the lack of bone marrow toxicity in some of the WT1 vaccinated patients who show tumor regression. It is possible that the immune response has been directed against a cryptic epitope, not present in healthy bone marrow cells.

Molldrem et al. provided evidence that tumor cells can shape the T-cell repertoire [19](reviewed in the context of AML in [2]). High avidity, proteinase 3 (PR1)-specific Tcells killed chronic myeloid leukemia (CML) cells more effectively than low-avidity T-cells but these cells became apoptotic when co-cultured with a high PR1 peptide concentration or with leukemia cells overexpressing proteinase 3. Furthermore, no high avidity T-cells could be detected in patients at initial presentation, whereas low avidity T-cells could be detected and expanded [19].

Many peptides derived from tumor antigens are characterized by weak binding to MHC molecules with a resulting insufficient stimulation of antitumor immunity. This hypothesis is supported by studies which have shown that the MHC binding affinity of peptides can correlate with immunogenicity [44]. Engels et al. demonstrated that a high-affinity peptideMHC (pMHC) interaction led to efficient cross-presentation and was necessary to elicit Tcell cytokine production in vivo [45]. A strong correlation between the high-affinity of the peptide for MHC and tumor eradication was described. Peptides with high affinities for MHC were effectively cross-presented by stromal cells, or cancer cells, and resulted in the secretion of IFN- $\gamma$ in cognate T-cells ex vivo but this was not the case when low affinity peptides were used. On the basis of these studies Engels et al stated that heteroclitic peptide vaccination will never work because although modified peptides may induce strong T-cell responses they will not be able to induce tumor irradication if the native tumor antigen has a low affinity for the MHC. In contrast, several groups have demonstrated that the immunogenicity of peptides depends on MHC-binding stability rather than affinity [46,47]. Therefore, low affinity, 
cryptic epitopes have been investigated for their potential as peptide vaccines. When supplied exogenously, such epitopes often fail to bind to MHC molecules for long enough, or at a high enough pMHC density, to efficiently stimulate $\mathrm{CD}^{+}{ }^{+} \mathrm{T}$-cells.

\section{Analogue peptides}

Another possibility to enhance the immunogenicity of LAAs in peptide vaccination studies is the use of analogue peptides with a change at one of the anchor positions. This method is based on the immunological synapse consisting of the MHC class I or class II complex, the epitope processed from the antigen, and presented on MHC, and the T-cell receptor (TCR). Costimulatory molecule signals ensure that the T-cells are activated and proliferate, in their absence T-cells become tolerant to the presented epitope despite the TCR binding to the pMHC. The greater the number of a given pMHC complex on the cell surface, the greater the chance of the corresponding TCR being engaged at the threshold level required to achieve activation. Peptides bind to MHC with different affinities depending on the constituent amino acids particularly at the anchor positions [47]. It has been shown that only one to two amino acids interact with the TCR and that its promiscuity allows one TCR to recognize numerous pMHC, thus allowing a single T-cell to be responsive to at least four distinct determinants within three different MHCs [48,49].

The stability of the pMHC and hence its effectiveness at stimulating an immune response can be altered by changing one of the anchor residues of the epitope sequence, amino acids in position 2 or position 9 in the case of HLA-A2 [50,51]. Such alterations to the anchor residues can enhance peptide immunogenicity [44] and success of this strategy is dependent on the TCR binding portion of the peptide not being altered significantly by anchor residue 
substitution. The fact that TCR recognition exhibits a degree of cross reactivity [52] makes this a viable approach. Peptides redesigned in this way and which induce effective T-cell responses are commonly referred to as 'heteroclitic peptides' or 'peptide analogues' and have shown efficacy in various tumor types, including chronic lymphocytic leukemia [53], CML [54] and AML [55].

Chen et al. demonstrated that an analogue peptide of NY-ESO-1-SLLMWITQC (wt), with a single change at the amino acid in position 9, had strengthened epitope binding to the MHC class I complex and enhanced TCR affinity in mice [56]. Another interesting observation in this study was that NY-ESO-1-wt specific CTLs from analogue peptide primed mice have an enhanced ability to recognize target cells pulsed with the wt peptide compared to wt-specific T-cells from wt-peptide primed mice.

Similar results have been reported in WT1 studies, whereby a modified WT1 peptide significantly enhanced the level of lysis of WT-1 expressing tumour cells by T-cells compared with wt peptide [57]. To circumvent tolerance, Pinilla-Ibarz et al tested analogue peptides for their ability to bind MHC class I and expand T-cells which could still cross-react with the native peptide and kill target CML cells. All peptides were made through a single amino acid substitution at the HLA-A*0201 binding site [58]. Subsequently, May et al. developed a WT1 CD4 ${ }^{+}$peptide epitope, 122-140, which was mutated through a substitution of residue 126 (arginine to tyrosine)[59]. The group demonstrated that native WT1 epitopes presented on the surface of human cancer cells could be recognized by CD4 ${ }^{+}$and WT1specific CTL; which were induced in response to the peptide analogue produced through the mutation of wt peptide. To date, most strategies have aimed to maximize the efficiency of peptide vaccines by focussing on pMHC binding, but the interaction between the TCR and pMHC complex can also be enhanced [60]. A variant of a CEA epitope, altered at the TCR 
contact site, has been reported to cause clinical responses in colorectal cancer patients [61] and in myeloid leukemia TCR modified to better recognize WT1 epitopes have shown promising results (recently reviewed in [62]).

\section{Modified peptides characterized for use in AML clinical trials}

Examples of LAA derived modified peptides which have shown promise include:

\section{CD33}

Bae et al. predicted a number of modified CD33 peptides with potentially enhanced immunogenicity $[63,64]$. Their modifications, in the form of single amino acid replacement, were designed to increase HLA-A*0201 or TCR affinity when compared to the native CD33 peptide. The studies concluded that the modified peptides were capable of producing highly cytotoxic CTLs, which were specific for both the native and modified peptide without inhibiting normal progenitor cells.

\section{WT1}

Patients whose leukemia cells showed an exceptionally high expression of WT1, exhibited a significantly poorer prognosis than those expressing lower levels [65], implying that WT1 is intimately associated with tumor progression and is therefore a particularly attractive target for the immunotherapy of leukemia. To date, several HLA-A*0201-binding peptides $\left(\mathrm{WT}_{37-45}, \mathrm{WT} 1_{126-134}, \mathrm{WT} 1_{187-194}\right.$ and $\left.\mathrm{WT} 1_{235-243}\right)$ have been identified $[11,66-68]$ and T-cell populations with specificity for each of these pMHC complexes have been found in AML and CML patients, as well as healthy individuals. The presence of T-cells with specificity for the WT1 $1_{126}$ epitope correlates with favourable prognostic indicators in HLA-A2 ${ }^{+}$patients with 
ALL and AML [69], and relatively recently this peptide was tested in a clinical setting [70]. One of these four epitopes (WT1 $\left.{ }_{235-243}\right)$ showed additional binding to HLA-A24, a haplotype common in the Far East. However, it was noted that the sequence of this peptide contained only one anchor residue for HLA-A24 binding; a lysine at position 9 [57]. By substituting a tyrosine for a methionine at position 2, a 28-fold increase in HLA-A24-binding of the analogue peptide, compared with the wt peptide, was obtained. Importantly, T-cells from healthy donors stimulated with the analogue peptide in vitro exhibited greater lysis of target cells expressing the wt peptide, compared with T-cells stimulated with the wt epitope. A phase I clinical study was subsequently initiated, in which HLA-A $24^{+}$patients with a range of cancers, including AML, were vaccinated with either the wt or the modified peptide delivered in increasing doses (0.3-3mg), administered with Montanide ISA51 adjuvant. Vaccination was found to be generally safe, although the two myelodysplastic syndrome patients included in the study exhibited leukocytopenia. In total, nine of the 13 patients showed an increase in WT1-tetramer positive T-cells following vaccination, including four of the five AML patients. It should be noted that the tetramer used in this study incorporated the wt peptide and the presence of tetramer ${ }^{+}$cells in patients immunized with the modified peptide, confirmed that T-cells expanded with the analogue peptide retained specificity for the wt peptide. The number of patients recruited to this study precludes meaningful comparisons between the efficacy of the analogue and wt peptide, although it should be noted that significant responses were observed in patients immunized with either peptide [71].

Identification of additional WT1-derived epitopes and modified peptide ligands continue in pre-clinical studies. Pinilla-Ibarz et al. [58] showed that a modified form of WT1 $126-134$, in which the first residue, an $\mathrm{R}$ (arginine), was replaced with a $\mathrm{Y}$ (tyrosine), exhibits similar binding to HLA-A2 as the wt epitope, yet is more efficient at expanding healthy HLA-A2 ${ }^{+} \mathrm{T}-$ 
cells in vitro, suggesting that this altered peptide ligand is heteroclitic. Importantly, altered peptide ligand-expanded T-cells lysed targets pulsed with wt peptide, as well as primary CML blasts. A modified version of $\mathrm{WT} 1_{187-195}$, in which the first residue (S, serine) was again substituted for a Y (tyrosine), exhibited improved binding to HLA-A2 and increased Tcell expansion. These expanded T-cells retained their ability to lyse cells expressing the wt peptide. Taken together, these results showed the difficulties incurred when predicting altered peptide ligands; a similar substitution in two peptides designed to bind to the same $\mathrm{MHC}$, in one case resulted in no change in MHC binding but expanded T-cells in vitro, while the other improved MHC binding and T-cell expansion. A panel of additional WT1-derived epitopes and altered peptide ligands were identified by this group, however these varied enormously in the availability of a suitable repertoire of T-cells, and in their efficacy at killing cells harbouring the wt peptide and/or an altered peptide ligand. The work highlighted the importance of functional assays to confirm the predictions made by algorithms.

In a pilot study Maslak et al. delivered a polyvalent vaccine to 10 patients with AML. The vaccine incorporated two long WT1 $\mathrm{CD}^{+}$peptides (WT1-427 long, WT1-331 long), the WT1-derived peptide (WT1-A1) to stimulate $\mathrm{CD}^{+}$responses and one modified peptide (WT1-122A1), which can stimulate both $\mathrm{CD}^{+}$and $\mathrm{CD} 8^{+}$responses. As with other studies involving modified peptide vaccines, positive correlations were seen between administration of the vaccine peptide and production of CTLs. In this case, $87.5 \%$ of patients showed a WT1-specific T-cell response [72].

\section{PASD1}

In 2013, a modified peptide for the SEREX-identified PASD1 CTA [55] was identified. The group had initially examined seven wt predicted epitopes for PASD1 which could bind HLA- 
A*0201 and had low similarity with other known eukaryotic proteins, but all candidates showed poor binding scores by SYFPEITHI or BioInformatics \& Molecular Analysis Section (BIMAS). T2 cells were used to determine the ability of peptides to bind HLA-A2 and stabilize it through the detection of HLA-A2 levels on the cell surface. All wt PASD1 peptides had negligible binding [55]. However, on modification of either the second or ninth amino acid to isoleucine, leucine or valine, there was a notable improvement in the binding of the analogue peptides. When inserted into pDOM-epitope DNA vaccines, the Pa14 analogue peptide was shown to stimulate IFN- $\gamma$ secreting wt PASD1 responsive T-cells, and induce effective CTL responses against endogenously expressing PASD1 positive myeloid leukemia cells. However, no detectable T-cell response was found when T-cells were primed with the wt parental epitope (Pw8).

\section{Combinations of multiple peptides}

A further vaccination concept to circumvent tumor variant escape is to combine multiple peptides, hence answering the issue of changing peptide expression in an evolving malignant cell population. Although the available data is derived from experience with solid tumors (detailed in the following paragraph), it encompasses approaches that could be also transferred to hematological malignancies.

Sampson et al. described the loss of target antigen expression in the majority of glioblastoma patients, with recurrence, after peptide vaccination with a 13 -amino acid peptide derived from the EGFRvIII mutation. Whether the results were due to a downregulation of the antigen, or an elimination of the tumor cells that expressed the antigen, remains unclear [72]. In a randomized phase II trial of two multi-peptide vaccines for melanoma in the adjuvant setting, 
one patient group received a vaccine containing a mixture of 12 peptides from melanocytic differentiation proteins and CTAs, compared with a group receiving a mixture of four melanocyte differentiation peptides [22]. It was shown that the 12-peptide vaccine induced a greater cumulative T-cell response than the four-peptide vaccine, as evaluated by ELISpot assays with lymphocytes isolated from both the peripheral blood and from the lymph node draining the injection site. The study also demonstrated that competition among peptides for MHC binding in polyvalent vaccines does not significantly inhibit T-cell induction or T-cell effector function. Another example of a successful multi-peptide vaccination clinical trial is a vaccine for renal cell cancer, IMA901, which consists of ten different tumor-associated peptides, that is now entering a phase III study [30].

Interestingly, the order in which wt and modified peptides are used to vaccinate mice has been shown to change the avidity of CTLs by increasing the frequency of low-avidity wt specific CTL when a wt peptide prime was followed by a high dose modified peptide boost [74].

\section{Mutated epitopes (neoantigens)}

Alongside the exploitation of analogue peptides with modified anchor residues for improved stability of pMHC complexes or TCR binding portions, use of peptides with mutated epitopes as an alternative approach for immunotherapy is becoming increasingly attractive. One advantage of neoantigens is that high-avidity T-cells remain available to kill tumor cells [75] as these proteins were not presented during T-cell maturation in the thymus. This is because neoantigens can progress tumor development and for most leukemia patients this likely happens much later in the patients' life. To date, modified peptides have been derived mainly 
from non-mutated proteins or their non-mutated segments, however in order to preserve and expand T-cell populations, which can respond to neo-antigens, it would be reasonable to examine and target peptides with naturally mutated epitopes, where mutations would be tumor-associated or even tumor-specific. One example is NPM1. As a prognostic marker in AML, the NPM $1^{\text {mut }}$ protein plays a special role. Interestingly, AML patients with an $N P M 1^{\text {mut }}$ belong to a favorable group in the WHO classification. Mutations in the NPMI gene are one of the most frequent single gene mutations in AML (25\%-30\%) and they predominantly occur in AML patients with a normal karyotype (45\%-60\%) [76]. Although several mutations of the NPMI gene exist in AML, more than $90 \%$ harbor the so-called A, B and D mutations [77], therefore the mutations are quite homogenous. It is now known that the NPM1 mutation dislocates the nuclear expression of wt protein into the cytoplasm and interferes with the ARF/p53 and NF-kB pathways [78].

The favorable prognosis of AML with $N P M I^{\text {mut }}$ led to the hypothesis that immune responses may contribute to the positive outcome for patients through the specific lysis of residual leukemic cells that bear the $N P M 1^{\text {mut }}$. It was shown that epitopes derived from the mutated regions of NPM1 induced specific $\mathrm{CD}^{+}$and $\mathrm{CD}^{+}$T-cell responses. Two HLA-A2 restricted epitopes induced high frequencies of specific $\mathrm{CD}^{+} \mathrm{T}$-cell responses in healthy volunteers and AML patients [79]. Furthermore, a survival analysis of $25 N P M 1^{\text {mut }}$ patients, comparing cases with or without specific CTL responses against $N P M 1^{\text {mut }}$ epitopes, suggested a better overall survival of patients with specific $N P M 1^{m u t}$ CTL responses [80].

In an AML patient with $N P M 1^{\text {mut }}$ and molecular relapse, a polyspecific CTL response against several known LAAs, including an epitope derived from $N P M 1^{\text {mut }}$, was demonstrated after pre-emptive donor lymphocyte infusion [81]. Especially when considering patients harboring a low tumor burden, represented by MRD without hematological relapse, this subgroup 
would be especially suited to $N P M 1^{\text {mut }}$ peptide vaccination to expand $N P M 1^{\text {mut }}$-specific $\mathrm{CD}^{+} \mathrm{T}$-cells and prolong remission or remove MRD. The use of analogue peptides in this setting has yet to be elucidated.

\section{MHC class II epitopes}

For leukemia antigens, most studies have focused on fixed anchor peptides rather than other sequence modifications likely due to the difficulties in successfully identifying modified epitopes which are immunogenic and stimulate T-cell responses against tumor bearing cells. In addition, the majority of research groups have chosen to study MHC class I binding epitopes rather than MHC class II, since the effector cells in this scenario are thought to be $\mathrm{CD}^{+} \mathrm{T}$ lymphocytes that recognize peptides presented on MHC class I. Peptides are more promiscuous in their binding to MHC class II molecules and far more is known about the preferred anchor residues of MHC class I than MHC class II molecules. It is clear that for the optimal induction of $\mathrm{CD}^{+} \mathrm{T}$-cell memory, $\mathrm{CD} 4^{+} \mathrm{T}$-cells are required, therefore, simply vaccinating with a minimal MHC class I-binding peptide, or its altered peptide ligand, has been shown to be unlikely to provide long-term benefits to patients. Strategies to activate $\mathrm{CD}^{+}$helper T-cells have been shown to be effective in the context of DNA vaccines in mouse models in which the first domain of tetanus toxin is used to stimulate $\mathrm{CD}^{+}$help $[55,82,83]$ and altered WT1 peptide ligands designed for MHC class II are also being explored [84].

\section{Peptide vaccination and immune checkpoint modulation}


The history of peptide vaccination using single peptides shows that the breakthrough is still pending. In the meantime immunotherapy with immune checkpoint inhibitors such as cytotoxic T-cell lymphocyte antigen 4 (CTLA-4) and programmed cell death protein 1 (PD1) have been very successful in different solid tumors especially in multiple melanoma [85-87]. CTLA-4 acts as a competitive inhibitor of CD28 signaling, which due to its enhanced affinity for CD80/CD86, acts as a "brake" to limit immune responses. In addition CTLA-4 acts directly on T-cells to inhibit them. PD-1 signaling inhibits proliferation, survival and effector functions (such as cytotoxicity and cytokine release) in CTLs and induces apoptosis in these cells. PD1 silences T-cell activity by binding to the ligands PDL1 and PDL2. These two ligands are upregulated in response to inflammatory cytokines. PDL1 expression is commonly upregulated in tumor cells and PD1 expression is upregulated in tumor-infiltrating lymphocytes. Interestingly, the existence of neoantigens, seen where there is high mutational load, leads to a better response to immune checkpoint inhibitors, perhaps because there are higher affinity T-cells available and these are specific for neoantigens due to a lack of tolerance $[88,89]$.

Until now, there has been no clinical trial conducted combining peptide vaccination with an immune checkpoint inhibitor in hematological malignancies. In stage IV melanoma, a study investigating a multiple MART-1 analog peptide vaccination, with or without IMP321 (a LAG-3Ig -lymphocyte activation gene- fusion protein), in combination with lymphodepleting chemotherapy and adoptive transfer of autologous PBMCs was performed. The vaccination in combination with the LAG-3Ig fusion protein, as an adjuvant, induced more robust and durable cellular antitumor immune responses [90]. A phase 3 study in patients with metastatic melanoma demonstrated that the addition of a glycoprotein 100 (gp100) peptide with ipilimumab (a CTLA-4 inhibitor) did not improve overall-survival any more 
than ipilimumab alone, and progression-free survival was even lower in the gp100 plus ipilimumab treated patients [85]. In contrast, the addition of interleukin 2 to gp100 led to a significant improvement in progression-free survival with a trend towards prolonged median overall-survival compared to interleukin 2 alone [91].

With the introduction of immune checkpoint inhibitors into the clinic plenty of new possibilities for immunotherapy exist. Further clinical trials to test the optimal immune checkpoint inhibitors in combination with the optimal LAA have a role to play in the future.

\section{Summary}

It is increasingly possible to determine the phenotype of cancer stem cells and their properties with regards to chemotherapy, radiotherapy and possibly immunotherapy resistance (reviewed in [92]). Much can be learnt about cancer stem cells from AML by virtue of it being a cancer of (hematopoietic) stem cells and it is hoped that ways to circumvent their resilience to treatment will improve survival rates in this difficult to treat disease.

Vaccines which stimulate specific immune responses against AML have been numerous in vitro and donor lymphocyte infusions are one immunotherapy strategy that has been shown to be very effective at improving patient response rates - extending and achieving durable remission. However this is a balancing act against the inevitable induction of Graft versus Host Disease (GvHD) which can be life-limiting in some patients [93].

Success of the strategy of modifying epitopes to enhance anti-cancer immune responses are dependent on the TCR binding portion of the peptide not being altered significantly by 
anchor residue substitution [44]. Similar modifications have led to peptide analogues which are effective at inducing immune responses against a range of tumor types including leukemia and solid tumors [54,57-59,94,95](reviewed in [96]). Despite inherent problems in demonstrating the ability of modified peptides to recognize and kill tumor cells, some heteroclitic epitopes have shown promise in phase I clinical trials $[18,71,97]$. Future studies will increasingly focus on mutated peptides that provide a cancer specific target for immunotherapy and the examination of overlapping peptide pools [98] to determine natural epitopes, that often exist at low frequencies, for future targeting. Undoubtedly, immunotherapy clinical trials will increasingly focus on multi-epitopes from within the same antigen or from different antigens in combination with immunomodulators such as CTLA4 or PD1 to enhance epitope spreading and minimize immune escape. This should enable the removal, or at the very least lead to a reduction in MRD during first remission, a state achieved by most patients with AML.

Of note $\mathrm{CD}^{+}$epitopes embedded within $\mathrm{CD} 4^{+}$recognized sequences offer the benefit of inducing $\mathrm{CD}^{+}$responses in the presence of much needed $\mathrm{CD} 4^{+}$help. In pDOM-epitope DNA vaccines $[55,82,83]$ this help is provided by the first domain of tetanus toxin which can induce $\mathrm{CD}^{+}$T-cell responses without subverting the tumor specificity of the vaccine. Translocation regions within chromosomes in AML cells may be targeted by miRNAs and siRNAs and provide the requisite specific targeting and AML-host cell destruction. Dendritic cells may also offer a powerful vehicle to present modified peptides to the immune system in AML patients [99]. Combination therapies which include chemotherapy to reduce tumor load and immunotherapy to remove MRD are expected to be superseded by conventional therapies used in conjunction with combination-immunotherapy protocols including the vaccination of patients with peptides following adoptive T-cell transfer [100] to remove residual disease. 
Immunotherapy and our understanding of peptide vaccines continues to require further investigation to help this therapy reach its full potential in the clinic, ideally as part of a personalized therapy plan. For cancers as heterogeneous as myeloid leukemia, the cure is likely to need a multi-faceted and increasingly patient specific focus to maximise survival rates.

\section{Acknowledgements}

We would like to thank Dr Sarah Buchan for her helpful insights. Dr Susanne Hofmann received funding from the German Research Foundation (Deutsche Forschungsgemeinschaft, DFG) and Drs Nicola Hardwick and Barbara Guinn from Leukaemia and Lymphoma Research.

\section{Conflict of interest}

The authors declare there are no competing financial interests in relation to the work described. 


\section{References}

1. Guinn BA, Mohamedali A, Thomas NS, Mills KI (2007) Immunotherapy of myeloid leukaemia. Cancer Immunol Immunother. 56:943-57.

2. Chan L, Hardwick NR, Guinn BA, Darling D, Gaken J, Galea-Lauri J, et al (2006) An immune edited tumour versus a tumour edited immune system: Prospects for immune therapy of acute myeloid leukaemia. Cancer Immunol Immunother. 55:1017-24.

3. Cheuk AT, Chan L, Czepulkowski B, Berger SA, Yagita H, Okumura K, et al (2006) Development of a whole cell vaccine for acute myeloid leukaemia. Cancer Immunol Immunother. 55:68-75.

4. van der Bruggen P, Traversari C, Chomez P, Lurquin C, De Plaen E, Van den Eynde $\mathrm{B}$, et al (1991) A gene encoding an antigen recognized by cytolytic $\mathrm{T}$ lymphocytes on a human melanoma. Science. 254:1643-7.

5. Sahin U, Tureci O, Schmitt H, Cochlovius B, Johannes T, Schmits R, et al (1995) Human neoplasms elicit multiple specific immune responses in the autologous host. Proc Natl Acad Sci U S A. 92:11810-3.

6. Cheever MA, Allison JP, Ferris AS, Finn OJ, Hastings BM, Hecht TT, et al (2009) The prioritization of cancer antigens: a national cancer institute pilot project for the acceleration of translational research. Clin Cancer Res. 15:5323-37.

7. Khan G, Brooks SE, Mills KI, Guinn BA. (2015) Expression of the cancer-testis antigen, PASD1, in ovarian cancer. Biomark Cancer. 7:31-8.

8. Adams SP, Sahota SS, Mijovic A, Czepulkowski B, Padua RA, Mufti GJ, et al (2002) Frequent expression of HAGE in presentation chronic myeloid leukaemias. Leukemia. 16:2238-42.

9. Van Driessche A, Berneman ZN, Van Tendeloo VF (2012) Active specific immunotherapy targeting the Wilms' tumor protein 1 (WT1) for patients with hematological malignancies and solid tumors: lessons from early clinical trials. Oncologist. 17:250-9.

10. Guinn BA, Bland EA, Lodi U, Liggins AP, Tobal K, Petters S, et al (2005) Humoral detection of leukaemia-associated antigens in presentation acute myeloid leukaemia. Biochem Biophys Res Commun. 335:1293-304.

11. Bellantuono I, Gao L, Parry S, Marley S, Dazzi F, Apperley J, et al (2002) Two distinct HLA-A0201-presented epitopes of the Wilms tumor antigen 1 can function as targets for leukemia-reactive CTL. Blood. 100:3835-7.

12. Kronke J, Schlenk RF, Jensen KO, Tschurtz F, Corbacioglu A, Gaidzik VI, et al (2011) Monitoring of minimal residual disease in NPM1-mutated acute myeloid leukemia: a study from the German-Austrian acute myeloid leukemia study group. J Clin Oncol. 29:2709-16.

13. Inoue K, Ogawa H, Sonoda Y, Kimura T, Sakabe H, Oka Y, et al (1997) Aberrant overexpression of the Wilms tumor gene (WT1) in human leukemia. Blood. 89:1405-12.

14. Brossart P, Wirths S, Stuhler G, Reichardt VL, Kanz L, Brugger W (2000) Induction of cytotoxic T-lymphocyte responses in vivo after vaccinations with peptide-pulsed dendritic cells. Blood. 96:3102-8.

15. Ochsenreither S, Fusi A, Busse A, Bauer S, Scheibenbogen C, Stather D, et al (2011) "Wilms Tumor Protein 1" (WT1) peptide vaccination-induced complete remission in a patient with acute myeloid leukemia is accompanied by the emergence of a predominant $\mathrm{T}$ cell clone both in blood and bone marrow. J Immunother.34:85-91.

16. Rezvani K, Yong AS, Mielke S, Savani BN, Musse L, Superata J, et al (2008) Leukemia-associated antigen-specific T-cell responses following combined PR1 and WT1 peptide vaccination in patients with myeloid malignancies. Blood. 111:236-42. 
17. Uttenthal B, Martinez-Davila I, Ivey A, Craddock C, Chen F, Virchis A, et al (2014) Wilms' Tumour 1 (WT1) peptide vaccination in patients with acute myeloid leukaemia induces short-lived WT1-specific immune responses. Br J Haematol. 164:366-75.

18. Rezvani K, Yong AS, Tawab A, Jafarpour B, Eniafe R, Mielke S, et al (2009) Ex vivo characterization of polyclonal memory CD8+ T-cell responses to PRAME-specific peptides in patients with acute lymphoblastic leukemia and acute and chronic myeloid leukemia. Blood. 113:2245-55.

19. Molldrem JJ, Lee PP, Kant S, Wieder E, Jiang W, Lu S, et al (2003) Chronic myelogenous leukemia shapes host immunity by selective deletion of high-avidity leukemiaspecific T cells. J Clin Invest. 111:639-47.

20. Qazilbash MH, Weider E, Rios R, Lu S, Kant S, Giralt S, Estey E, Thall P, de Lima M, Couriel D, Champlin RE, Komanduri K, Molldrem JJ (2004) Vaccination with the PR1 leukemia-associated antigen can induce complete remission in patients with myeloid leukemia. Blood (ASH Annual Meeting Abstracts). 104:abstract 259.

21. Bijker MS, van den Eeden SJ, Franken KL, Melief CJ, Offringa R, van der Burg SH (2007) CD8+ CTL priming by exact peptide epitopes in incomplete Freund's adjuvant induces a vanishing CTL response, whereas long peptides induce sustained CTL reactivity. J Immunol.179:5033-40.

22. Slingluff CL, Jr., Petroni GR, Chianese-Bullock KA, Smolkin ME, Hibbitts S, Murphy C, et al (2007) Immunologic and clinical outcomes of a randomized phase II trial of two multipeptide vaccines for melanoma in the adjuvant setting. Clin Cancer Res. 13:638695.

23. Wells JW, Cowled CJ, Farzaneh F, Noble A (2008) Combined triggering of dendritic cell receptors results in synergistic activation and potent cytotoxic immunity. J Immunol. 181:3422-31.

24. Kochenderfer JN, Simpson JL, Chien CD, Gress RE (2007) Vaccination regimens incorporating CpG-containing oligodeoxynucleotides and IL-2 generate antigen-specific antitumor immunity from $\mathrm{T}$-cell populations undergoing homeostatic peripheral expansion after BMT. Blood. 110:450-60.

25. Suekane S, Nishitani M, Noguchi M, Komohara Y, Kokubu T, Naitoh M, et al (2007) Phase I trial of personalized peptide vaccination for cytokine-refractory metastatic renal cell carcinoma patients. Cancer Sci. 98:1965-8.

26. Purcell AW, McCluskey J, Rossjohn J (2007) More than one reason to rethink the use of peptides in vaccine design. Nat Rev Drug Discov. 6:404-14.

27. Speiser DE, Lienard D, Rufer N, Rubio-Godoy V, Rimoldi D, Lejeune F, et al (2005) Rapid and strong human CD8+ T cell responses to vaccination with peptide, IFA, and CpG oligodeoxynucleotide 7909. J Clin Invest. 115:739-46.

28. Lovgren T, Baumgaertner P, Wieckowski S, Devevre E, Guillaume P, Luescher I, et al (2012) Enhanced cytotoxicity and decreased CD8 dependence of human cancer-specific cytotoxic $\mathrm{T}$ lymphocytes after vaccination with low peptide dose. Cancer Immunol Immunother. 61:817-26.

29. Estey E, Dohner H. Acute myeloid leukaemia. Lancet. 2006;368(9550):1894-907.

30. Walter S, Weinschenk T, Stenzl A, Zdrojowy R, Pluzanska A, Szczylik C, et al (2012) Multipeptide immune response to cancer vaccine IMA901 after single-dose cyclophosphamide associates with longer patient survival. Nat Med. 18:1254-61.

31. Ghiringhelli F, Menard C, Puig PE, Ladoire S, Roux S, Martin F, et al (2007) Metronomic cyclophosphamide regimen selectively depletes CD4+CD25+ regulatory $\mathrm{T}$ cells and restores $\mathrm{T}$ and $\mathrm{NK}$ effector functions in end stage cancer patients. Cancer Immunol Immunother. 56:641-8. 
32. Sevko A, Sade-Feldman M, Kanterman J, Michels T, Falk CS, Umansky L, et al (2013) Cyclophosphamide promotes chronic inflammation-dependent immunosuppression and prevents antitumor response in melanoma. J Investig Dermatol. 133:1610-9.

33. Serrano A, Tanzarella S, Lionello I, Mendez R, Traversari C, Ruiz-Cabello F, et al (2001) Rexpression of HLA class I antigens and restoration of antigen-specific CTL response in melanoma cells following 5-aza-2'-deoxycytidine treatment. Int J Cancer. 94:243-51.

34. Keskinen P, Ronni T, Matikainen S, Lehtonen A, Julkunen I (1997) Regulation of HLA class I and II expression by interferons and influenza A virus in human peripheral blood mononuclear cells. Immunology 91:421-9.

35. Chang DH, Liu N, Klimek V, Hassoun H, Mazumder A, Nimer SD, et al (2006) Enhancement of ligand-dependent activation of human natural killer T cells by lenalidomide: therapeutic implications. Blood. 108:618-21.

36. Ko JS, Zea AH, Rini BI, Ireland JL, Elson P, Cohen P, et al (2009) Sunitinib mediates reversal of myeloid-derived suppressor cell accumulation in renal cell carcinoma patients. Clin Cancer Res. 15:2148-57.

37. Cibotti R, Kanellopoulos JM, Cabaniols JP, Halle-Panenko O, Kosmatopoulos K, Sercarz E, et al. (1992) Tolerance to a self-protein involves its immunodominant but does not involve its subdominant determinants. Proc Natl Acad Sci U S A. 89:416-20.

38. Warnock MG, Goodacre JA (1997) Cryptic T-cell epitopes and their role in the pathogenesis of autoimmune diseases. Br J Rheumatol. 36:1144-50.

39. Scardino A, Gross DA, Alves P, Schultze JL, Graff-Dubois S, Faure O, et al (2002) HER-2/neu and hTERT cryptic epitopes as novel targets for broad spectrum tumor immunotherapy. J Immunol. 168:5900-6.

40. Maecker B, Sherr DH, Vonderheide RH, von Bergwelt-Baildon MS, Hirano N, Anderson KS, et al (2003) The shared tumor-associated antigen cytochrome P450 1B1 is recognized by specific cytotoxic T cells. Blood. 102:3287-94.

41. Maecker B, von Bergwelt-Baildon MS, Sherr DH, Nadler LM, Schultze JL (2005) Identification of a new HLA-A*0201-restricted cryptic epitope from CYP1B1. Int J Cancer. 115:333-6.

42. Pascolo S, Bervas N, Ure JM, Smith AG, Lemonnier FA, Perarnau B (1997) HLAA2.1-restricted education and cytolytic activity of CD8(+) $\mathrm{T}$ lymphocytes from beta2 microglobulin (beta2m) HLA-A2.1 monochain transgenic $\mathrm{H}-2 \mathrm{Db}$ beta2m double knockout mice. J Exp Med. 185:2043-51.

43. Gross DA, Graff-Dubois S, Opolon P, Cornet S, Alves P, Bennaceur-Griscelli A, et al (2004) High vaccination efficiency of low-affinity epitopes in antitumor immunotherapy. J Clin Invest. 113:425-33.

44. Chen JL, Stewart-Jones G, Bossi G, Lissin NM, Wooldridge L, Choi EM, et al. (2005) Structural and kinetic basis for heightened immunogenicity of T cell vaccines. J Exp Med. 201:1243-55.

45. Engels B, Engelhard VH, Sidney J, Sette A, Binder DC, Liu RB, et al (2013) Relapse or eradication of cancer is predicted by peptide-major histocompatibility complex affinity. Cancer Cell. 23:516-26.

46. van der Burg SH, Visseren MJ, Brandt RM, Kast WM, Melief CJ (1996) Immunogenicity of peptides bound to MHC class I molecules depends on the MHC-peptide complex stability. J Immunol. 156:3308-14.

47. Harndahl M, Rasmussen M, Roder G, Dalgaard Pedersen I, Sorensen M, Nielsen M, et al (2012) Peptide-MHC class I stability is a better predictor than peptide affinity of CTL immunogenicity. Eur J Immunol. 42:1405-16. 
48. Mason D (1998) A very high level of crossreactivity is an essential feature of the Tcell receptor. Immunol Today. 19:395-404.

49. Bhardwaj V, Kumar V, Geysen HM, Sercarz EE (1993) Degenerate recognition of a dissimilar antigenic peptide by myelin basic protein-reactive $T$ cells. Implications for thymic education and autoimmunity. J Immunol. 151:5000-10.

50. Bakker AB, van der Burg SH, Huijbens RJ, Drijfhout JW, Melief CJ, Adema GJ, et al. (1997) Analogues of CTL epitopes with improved MHC class-I binding capacity elicit anti-melanoma CTL recognizing the wild-type epitope. Int J Cancer. 70:302-9.

51. Parkhurst MR, Salgaller ML, Southwood S, Robbins PF, Sette A, Rosenberg SA, et al (1996) Improved induction of melanoma-reactive CTL with peptides from the melanoma antigen gp100 modified at HLA-A*0201-binding residues. J Immunol. 157:2539-48.

52. Frankild S, de Boer RJ, Lund O, Nielsen M, Kesmir C (2008) Amino acid similarity accounts for $\mathrm{T}$ cell cross-reactivity and for "holes" in the $\mathrm{T}$ cell repertoire. PLoS One. 3:e1831.

53. Zirlik KM, Zahrieh D, Neuberg D, Gribben JG (2006) Cytotoxic T cells generated against heteroclitic peptides kill primary tumor cells independent of the binding affinity of the native tumor antigen peptide. Blood. 108:3865-70.

54. Pinilla-Ibarz J, Korontsvit T, Zakhaleva V, Roberts W, Scheinberg DA (2005) Synthetic peptide analogs derived from bcr/abl fusion proteins and the induction of heteroclitic human T-cell responses. Haematologica. 90:1324-32.

55. Hardwick N, Buchan S, Ingram W, Khan G, Vittes G, Rice J, et al. (2013) An analogue peptide from the Cancer/Testis antigen PASD1 induces CD8+ T cell responses against naturally processed peptide. Cancer Immunity. 13:16.

56. Chen JL, Dunbar PR, Gileadi U, Jager E, Gnjatic S, Nagata Y, et al. (2000) Identification of NY-ESO-1 peptide analogues capable of improved stimulation of tumorreactive CTL. J Immunol. 165:948-55.

57. Tsuboi A, Oka Y, Udaka K, Murakami M, Masuda T, Nakano A, et al (2002) Enhanced induction of human WT1-specific cytotoxic T lymphocytes with a 9-mer WT1 peptide modified at HLA-A*2402-binding residues. Cancer Immunol Immunother. 51:61420.

58. Pinilla-Ibarz J, May RJ, Korontsvit T, Gomez M, Kappel B, Zakhaleva V, et al (2006) Improved human T-cell responses against synthetic HLA-0201 analog peptides derived from the WT1 oncoprotein. Leukemia. 20:2025-33.

59. May RJ, Dao T, Pinilla-Ibarz J, Korontsvit T, Zakhaleva V, Zhang RH, et al (2007) Peptide epitopes from the Wilms' tumor 1 oncoprotein stimulate CD4+ and CD8+ T cells that recognize and kill human malignant mesothelioma tumor cells. Clin Cancer Res. 13:4547-55.

60. Meng WS, Butterfield LH (2002) Rational design of peptide-based tumor vaccines. Pharm Res. 19:926-32.

61. Fong L, Hou Y, Rivas A, Benike C, Yuen A, Fisher GA, et al (2001) Altered peptide ligand vaccination with Flt3 ligand expanded dendritic cells for tumor immunotherapy. Proc Natl Acad Sci U S A. 98:8809-14.

62. Stauss HJ, Morris EC (2013) Immunotherapy with gene-modified T cells: limiting side effects provides new challenges. Gene Therapy. 20:1029-32.

63. Bae J, Martinson JA, Klingemann HG (2004) Heteroclitic CD33 peptide with enhanced anti-acute myeloid leukemic immunogenicity. Clin Cancer Res. 10:7043-52.

64. Bae J, Martinson JA, Klingemann HG (2004) Identification of novel CD33 antigenspecific peptides for the generation of cytotoxic $\mathrm{T}$ lymphocytes against acute myeloid leukemia. Cellular Immunol. 227:38-50. 
65. Inoue K, Sugiyama H, Ogawa H, Nakagawa M, Yamagami T, Miwa H, et al (1994) WT1 as a new prognostic factor and a new marker for the detection of minimal residual disease in acute leukemia. Blood. 84:3071-9.

66. Gao L, Bellantuono I, Elsasser A, Marley SB, Gordon MY, Goldman JM, et al (2000) Selective elimination of leukemic CD34(+) progenitor cells by cytotoxic T lymphocytes specific for WT1. Blood. 95:2198-203.

67. Ohminami H, Yasukawa M, Fujita S (2000) HLA class I-restricted lysis of leukemia cells by a CD8(+) cytotoxic T-lymphocyte clone specific for WT1 peptide. Blood. 95:286-93. 68. Smithgall M, Misher L, Spies G, Cheever MA, Gaiger A (2001) Identification of a novel WT1 HLA-A*0201-restricted CTL epitope using whole gene in vitro priming [abstract]. American Society of Hematology Meeting; December 8-11, 2001; Orlando, FL. 69. Kapp M, Stevanović S, Fick K, Tan SM, Loeffler J, Opitz A, Tonn T, Stuhler G, Einsele H, Grigoleit GU. (2009) CD8+ T-cell responses to tumor-associated antigens correlate with superior relapse-free survival after allo-SCT. Bone Marrow Transplant. 43:399-410.

70. Keilholz U, Letsch A, Busse A, Asemissen AM, Bauer S, Blau IW, et al (2009) A clinical and immunologic phase 2 trial of Wilms tumor gene product 1 (WT1) peptide vaccination in patients with AML and MDS. Blood. 113:6541-8.

71. Oka Y, Tsuboi A, Taguchi T, Osaki T, Kyo T, Nakajima H, et al (2004) Induction of WT1 (Wilms' tumor gene)-specific cytotoxic T lymphocytes by WT1 peptide vaccine and the resultant cancer regression. Proc Natl Acad Sci U S A. 101:13885-90.

72. Maslak PG, Dao T, Krug LM, Chanel S, Korontsvit T, Zakhaleva V, et al (2010) Vaccination with synthetic analog peptides derived from WT1 oncoprotein induces T-cell responses in patients with complete remission from acute myeloid leukemia. Blood. 116:1719.

73. Sampson JH, Heimberger AB, Archer GE, Aldape KD, Friedman AH, Friedman HS, et al (2010) Immunologic escape after prolonged progression-free survival with epidermal growth factor receptor variant III peptide vaccination in patients with newly diagnosed glioblastoma. J Clin Oncol. 28:4722-9.

74. Narayan S, Choyce A, Fernando GJ, Leggatt GR (2007) Secondary immunisation with high-dose heterologous peptide leads to CD8 T cell populations with reduced functional avidity. Eur J Immunol. 37:406-15.

75. Gros A, Robbins PF, Yao X, Li YF, Turcotte S, Tran E, et al (2014) PD-1 identifies the patient-specific $\mathrm{CD} 8(+)$ tumor-reactive repertoire infiltrating human tumors. J Clin Invest. 124:2246-59.

76. Schlenk RF, Dohner K, Krauter J, Frohling S, Corbacioglu A, Bullinger L, et al (2008) Mutations and treatment outcome in cytogenetically normal acute myeloid leukemia. N Engl J Med. 358:1909-18.

77. Falini B, Nicoletti I, Bolli N, Martelli MP, Liso A, Gorello P, et al (2007) Translocations and mutations involving the nucleophosmin (NPM1) gene in lymphomas and leukemias. Haematologica. 92:519-32.

78. Cilloni D, Messa F, Rosso V, Arruga F, Defilippi I, Carturan S, et al. (2008) Increase sensitivity to chemotherapeutical agents and cytoplasmatic interaction between NPM leukemic mutant and NF-kappaB in AML carrying NPM1 mutations. Leukemia. 2008;22:1234-40.

79. Greiner J, Ono Y, Hofmann S, Schmitt A, Mehring E, Gotz M, et al (2012) Mutated regions of nucleophosmin 1 elicit both $\mathrm{CD} 4(+)$ and $\mathrm{CD} 8(+) \mathrm{T}$-cell responses in patients with acute myeloid leukemia. Blood. 120:1282-9.

80. Greiner J, Schneider V, Schmitt M, Gotz M, Dohner K, Wiesneth M, et al (2013) Immune responses against the mutated region of cytoplasmatic NPM1 might contribute to the 
favorable clinical outcome of AML patients with NPM1 mutations (NPM1mut). Blood. 122:1087-8.

81. Hofmann S, Gotz M, Schneider V, Guillaume P, Bunjes D, Dohner H, et al (2013) Donor lymphocyte infusion induces polyspecific CD8(+) T-cell responses with concurrent molecular remission in acute myeloid leukemia with NPM1 mutation. J Clin Oncol. 31:e44-7. 82. Chaise C, Buchan SL, Rice J, Marquet J, Rouard H, Kuentz M, et al (2008) DNA Vaccination Induces Wt1-Specific T-Cell Responses with Potential Clinical Relevance. Blood. 112:2956-64.

83. Padua RA, Larghero J, Robin M, le Pogam C, Schlageter MH, Muszlak S, et al (2003) PML-RARA-targeted DNA vaccine induces protective immunity in a mouse model of leukemia. Nature Med. 9:1413-7.

84. Kobayashi H, Nagato T, Aoki N, Sato K, Kimura S, Tateno M, et al (2006) Defining MHC class II T helper epitopes for WT1 tumor antigen. Cancer Immunol Immunother. 55:850-60.

85. Hodi FS, O'Day SJ, McDermott DF, Weber RW, Sosman JA, Haanen JB, et al (2010) Improved survival with ipilimumab in patients with metastatic melanoma. $\mathrm{N}$ Engl $\mathrm{J}$ Med. 363:711-23.

86. Topalian SL, Hodi FS, Brahmer JR, Gettinger SN, Smith DC, McDermott DF, et al (2012) Safety, activity, and immune correlates of anti-PD-1 antibody in cancer. N Engl J Med. 366:2443-54.

87. Hamid O, Robert C, Daud A, Hodi FS, Hwu WJ, Kefford R, et al (2013) Safety and tumor responses with lambrolizumab (anti-PD-1) in melanoma. N Engl J Med. 369:134-44.

88. Gubin MM, Zhang X, Schuster H, Caron E, Ward JP, Noguchi T, et al (2014) Checkpoint blockade cancer immunotherapy targets tumour-specific mutant antigens. Nature. 515:577-81.

89. Snyder A, Makarov V, Merghoub T, Yuan J, Zaretsky JM, Desrichard A, et al (2014) Genetic basis for clinical response to CTLA-4 blockade in melanoma. N Engl J Med. 371:2189-99.

90. Romano E, Michielin O, Voelter V, Laurent J, Bichat H, Stravodimou A, et al (2014) MART-1 peptide vaccination plus IMP321 (LAG-3Ig fusion protein) in patients receiving autologous PBMCs after lymphodepletion: results of a Phase I trial. J Transl Med. 12:97.

91. Schwartzentruber DJ, Lawson DH, Richards JM, Conry RM, Miller DM, Treisman J, et al (2011) gp100 peptide vaccine and interleukin-2 in patients with advanced melanoma. $\mathrm{N}$ Engl J Med. 364:2119-27.

92. Maccalli C, De Maria R (2015) Cancer stem cells: perspectives for therapeutic targeting. Cancer Immunol Immunother. 64:91-7

93. Kolb HJ, Schattenberg A, Goldman JM, Hertenstein B, Jacobsen N, Arcese W, et al (1995) Graft-versus-leukemia effect of donor lymphocyte transfusions in marrow grafted patients. Blood. 86:2041-50.

94. Christensen O, Lupu A, Schmidt S, Condomines M, Belle S, Maier A, et al (2009) Melan-A/MART1 analog peptide triggers anti-myeloma T-cells through crossreactivity with HM1.24. J Immunother. 32:613-21.

95. Fourcade J, Kudela P, Andrade Filho PA, Janjic B, Land SR, Sander C, et al (2008) Immunization with analog peptide in combination with $\mathrm{CpG}$ and montanide expands tumor antigen-specific CD8+ T cells in melanoma patients. J Immunother. 31:781-91.

96. Trajanoski Z, Maccalli C, Mennonna D, Casorati G, Parmiani G, Dellabona P (2015) Somatically mutated tumor antigens in the quest for a more efficacious patient-oriented immunotherapy of cancer. Cancer Immunol Immunother. 64:99-104 
97. Maslak PG, Dao T, Gomez M, Chanel S, Packin J, Korontsvit T, et al (2008) A pilot vaccination trial of synthetic analog peptides derived from the BCR-ABL breakpoints in CML patients with minimal disease. Leukemia. 22:1613-6.

98. Singh SK, Meyering M, Ramwadhdoebe TH, Stynenbosch LF, Redeker A, Kuppen PJ, et al (2012) The simultaneous ex vivo detection of low-frequency antigen-specific CD4+ and CD8+ T-cell responses using overlapping peptide pools. Cancer Immunol Immunother. 61:1953-63.

99. Subklewe M, Geiger C, Lichtenegger FS, Javorovic M, Kvalheim G, Schendel DJ, et al (2014) New generation dendritic cell vaccine for immunotherapy of acute myeloid leukemia. Cancer Immunol Immunother. 63:1093-103.

100. Ly LV, Sluijter M, Versluis M, Luyten GP, van Stipdonk MJ, van der Burg SH, et al (2010) Peptide vaccination after T-cell transfer causes massive clonal expansion, tumor eradication, and manageable cytokine storm. Cancer Res. 70:8339-46. 